\title{
Binding of glutamate to the umami receptor
}

\author{
J.J. López Cascales ${ }^{\text {a,c,d }}$, S.D. Oliveira Costa ${ }^{\text {a }}$, Bert L. de Groot ${ }^{\text {b }}$, D. Eric Walters ${ }^{\text {c,d,* }}$ \\ a Universidad Politécnica de Cartagena, Bioinformatic and Macromolecules Group (BIOMAC), Aulario II, Campus de Alfonso XIII, 30203 Cartagena, Murcia, Spain \\ b Max Planck Institute for Biophysical Chemistry, Computational Biomolecular Dynamics Group, Am Fassberg 11, 37077 Goettingen, Germany \\ c Department of Biochemistry and Molecular Biology, Chicago Medical School, Rosalind Franklin University of Medicine and Science, North Chicago, IL 60064, USA \\ d Department of Pharmaceutical Sciences, College of Pharmacy, Rosalind Franklin University of Medicine and Science, North Chicago, IL 60064, USA
}

\section{A R T I C L E I N F O}

\section{Article history:}

Received 28 July 2010

Received in revised form 13 September 2010

Accepted 15 September 2010

Available online 26 September 2010

\section{Keywords:}

Glutamate

Umami

Taste receptor

MD simulations

\begin{abstract}
A B S T R A C T
The umami taste receptor is a heterodimer composed of two members of the T1R taste receptor family: T1R1 and T1R3. It detects glutamate in humans, and is a more general amino acid detector in other species. We have constructed homology models of the ligand binding domains of the human umami receptor (based on crystallographic structures of the metabotropic glutamate receptor of the central nervous system). We have carried out molecular dynamics simulations of the ligand binding domains, and we find that the likely conformation is that T1R1 receptor protein exists in the closed conformation, and T1R3 receptor in the open conformation in the heterodimer. Further, we have identified the important binding interactions and have made an estimate of the relative free energies associated with the two glutamate binding sites.
\end{abstract}

(c) 2010 Elsevier B.V. All rights reserved.

\section{Introduction}

The umami taste receptor is a Class C G protein coupled receptor (GPCR [1]). In humans, it responds to L-glutamate and, to some extent, L-aspartate; in other species, it serves as a more general detector of L-amino acids in the diet [2]. The receptor is a heterodimer composed of the T1R1 and T1R3 members of the T1R family.

The T1R taste receptors are related to the metabotropic glutamate receptors ( $\mathrm{mGluR}$ ) of the central nervous system as well as to other Class C GPCR receptors. The Class C GPCR family has seven transmembrane helical segments, like all GPCRs. In addition, these receptors have a large $\mathrm{N}$-terminal ligand binding region (>500 amino acids) with a "clamshell" or "venus flytrap" fold, having two ligand binding domains that can open or close. This N-terminal region is linked to the transmembrane segment by a smaller ( 70 amino acids) cysteine-rich domain [3]. Class C GPCRs may function as homodimers or heterodimers.

$\mathrm{X}$-Ray crystallographic studies of the ligand binding region of some mGluRs with and without bound glutamate show that these domains can exist in both "open" and "closed" conformations [4-6]. In the unliganded form, both of these domains are in an open conformation; ligand binding stabilizes a "closed-open/active" state [4]. In this state, one protomer binds glutamate in the closed conformation, and the other binds glutamate in the open conformation. In addition, the dimer interface reorganizes in such a way that the angle between the monomers is decreased. This is illustrated schematically in Fig. 1.

\footnotetext{
* Corresponding author. Department of Biochemistry and Molecular Biology, Chicago Medical School, Rosalind Franklin University of Medicine and Science, North Chicago, IL 60064, USA. Tel.: + 1847578 8613; fax: + 18475783240.

E-mail address: Eric.Walters@RosalindFranklin.edu (D.E. Walters).
}

Homology modeling of the closely related sweet taste receptors (T1R2 + T1R3) has facilitated an understanding of the interactions of sweeteners with their receptor [7-12].

During the last two decades, the technique of molecular dynamics simulation (MD) has emerged as a valuable tool to provide information related to the dynamic and static properties of biophysical systems with atomic detail. Two factors have contributed to this: improvements in simulation algorithms, and significant improvement in computing power, permitting simulation of larger systems for longer trajectory times [13].

Here we report homology modeling of the ligand binding domain of the umami receptor, and molecular dynamics based evaluation of the binding of glutamate to the two likely binding sites. Recently, Zhang et al. [14] have modeled the binding of glutamate to T1R1. However, it is known that metabotropic glutamate receptors bind two molecules of glutamate, one in each protomer, and that these exhibit negative cooperativity [15]. Cooperativity has been postulated for the T1R family taste receptors as well [16]. Therefore we have modeled the ligand binding domains of the umami receptor with a glutamate molecule bound in each monomer. This approach should provide useful information about a possible second binding site, although it is unlikely that simulations can be run long enough to provide details about cooperativity.

\section{Methods}

\subsection{Setting up the molecular structures}

All molecular modeling was carried out using Molecular Operating Environment (MOE, version 2007.09, Chemical Computing Group, 


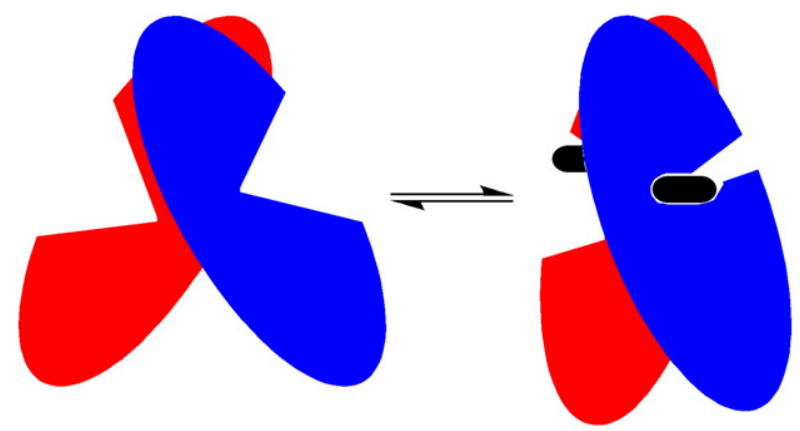

Fig. 1. Schematic representation of the ligand binding domains of glutamate receptors. Left, the unliganded "open-open/resting" state, in which both monomers have open conformations. Right, the liganded "closed-open/active" state, stabilized by glutamate binding, in which one monomer has a closed conformation, and the interface between monomers has rearranged so as to decrease the angle between the monomers.

Montreal). Homology modeling of the ligand binding region of the umami receptor was carried out as described previously for the sweet taste receptor [12], using the "closed-open/active" state of mGluR1 as the template (PDB code 1EWK [4]). The ligand binding domain of the mGluR1 has $26.8 \%$ sequence identity with human T1R1 and $24.1 \%$ identity with human T1R3. The sequence alignments used are included as Supplementary data. Homology modeling was carried out with all histidines in the protonated state.

The template structure is a homodimer, with the two chains adopting two different conformations, so we generated two models for the umami receptor: Form 1 has T1R1 in the closed conformation and T1R3 in the open conformation, and Form 2 has T1R1 in the open conformation and T1R3 in the closed conformation. The template structure has a disordered segment, residues 125-153, located near the top of the upper "clamshell" [4]. This segment contains a cysteine residue (Cys140 in mGluR1) that is known to form a disulfide linkage between the two subunits. In the T1R family, this region contains a cysteine (Cys129) in T1R3, but T1R1 and T1R2 do not have a cysteine in this region, so it was not necessary to model such a disulfide. Since the disordered regions are at least $30 \AA$ from the binding sites, we consider that they are unlikely to have a significant impact on calculated binding interactions.

The template structure includes a glutamate bound to each subunit, so we initially considered those two glutamate orientations. We also wished to account for the possibility of glutamate binding conformations different from those of the mGluR crystal structure, so alternate glutamate binding orientations were generated using the Dock module of MOE. First, a stochastic search method was used to generate a database of 220 L-glutamate conformations. Then the alpha-triangle method implemented in MOE was used to produce a series of docked poses in each binding site. Finally, minimization was carried out to produce starting points for molecular dynamics simulations. Ultimately, the starting points with the greatest number of favorable interactions with the binding sites corresponded to those of the template crystal structure.

\subsection{Setting up the MD simulations}

Two different systems were simulated in this study:

1. Form 1 in the presence of glutamate at both binding sites.

2. Form 2 in the presence of glutamate at both binding sites.

The starting Form 1 and Form 2 conformations obtained as described earlier were introduced into a three dimensional periodical computational box, and hydrated with a layer of water $1 \mathrm{~nm}$ thick, using a box containing 216 equilibrated SPC water molecules [17]. Thus, after solvating the proteins, the total number of atoms of the systems 1 and 2 was 95,682 and 92,161, respectively. To balance the positive charge of the systems, 2 chloride ions were introduced into the system by substitution of 2 water molecules in both cases. No other salt ions were included in the simulations. Thus, the final number of atoms of the systems was 95,676 and 92,115, respectively. The PDB files corresponding to the two starting conformations of the two systems are available from the corresponding author.

GROMACS 3.3.3 was the engine for all of the MD simulations $[18,19]$ and the OPLS force field [20] implemented in GROMACS was the force field used in all simulations. Once the starting configurations were generated as described earlier, the systems were subjected to a steepest descent minimization process to remove any existing strain or overlap between neighboring atoms. A time step of $2 \mathrm{fs}$ was used in all of the simulations. Due to the fact that all the simulations were performed under the NPT thermodynamic conditions, the systems were coupled to an isotropic external pressure and temperature bath of $1 \mathrm{~atm}$ and $298 \mathrm{~K}$, using Berendsen's algorithm [21]. The temperature and pressure constants used in our simulations were 0.1 and $0.5 \mathrm{ps}$ respectively. All the bond lengths in the system were constrained using LINCS [22]. Steric interactions were modeled with the Lennard-Jones potential, with a cut-off of $0.8 \mathrm{~nm}$, and electrostatic interactions were modeled with the Ewald algorithm [23,24].

Once the systems were set up, MD simulations of 100 ns each were carried out. To determine when the system had achieved an equilibrated state, the pair distances between glutamate and receptor were followed as a function of time. Fig. 2 corresponding to the distance between glutamate and binding site in T1R1 (system 1) shows that $50 \mathrm{~ns}$ of simulations are required to equilibrate the system. Similar results were seen for the other system (data not shown).

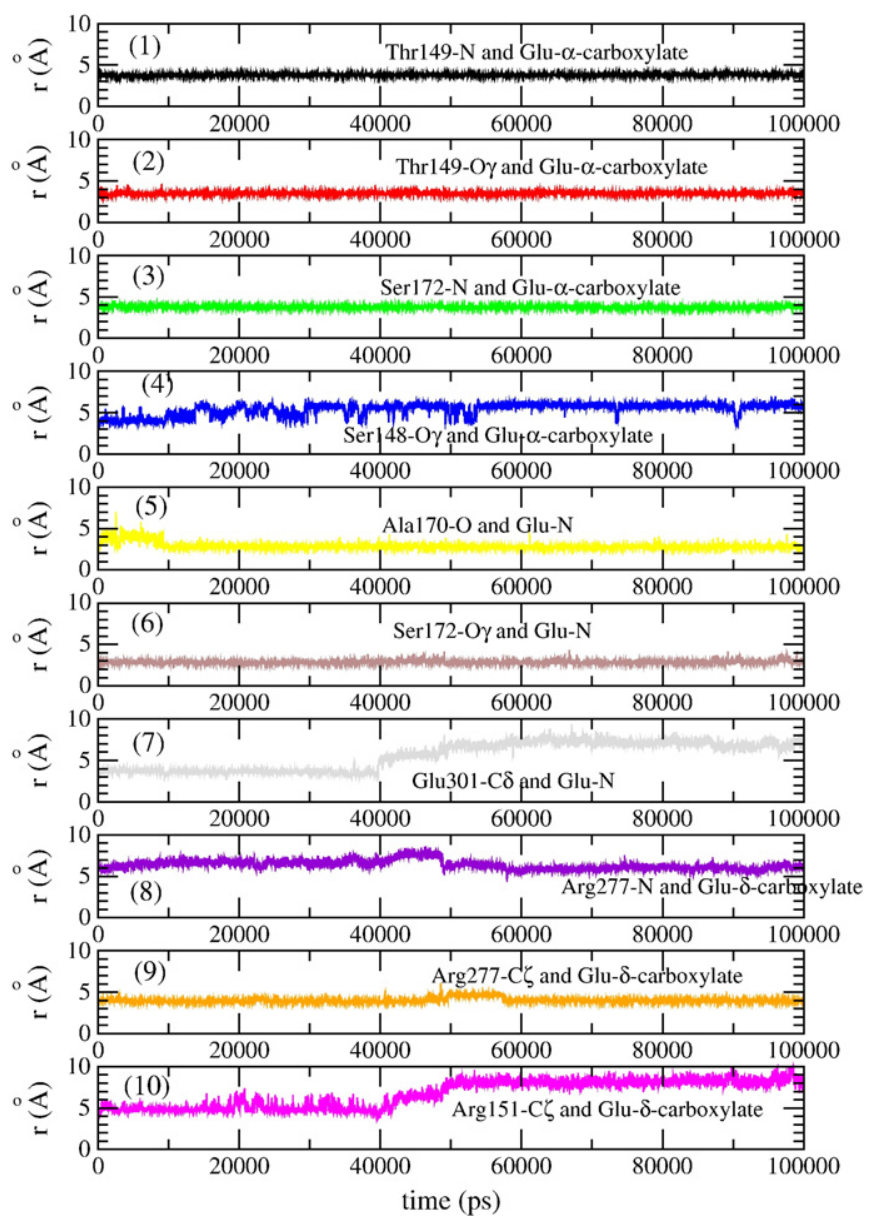

Fig. 2. Distances between Glutamate and T1R1 residues in binding site 1. 
Therefore, the first $50 \mathrm{~ns}$ of each simulated trajectory was omitted from further analysis in the present work.

\section{Results and discussion}

\subsection{Receptor conformation}

The mGluR1 template structure is a homodimer, while the umami receptor is a heterodimer. Since the active form is asymmetric, we first considered the two possible models: Form 1, in which T1R1 is in the closed conformation and T1R3 is in the open conformation, and Form 2 , in which T1R1 is in the open conformation and T1R3 is in the closed conformation. Our molecular dynamics simulations provide good evidence that Form 1 is the correct choice. Over the course of the 100 ns simulation of Form 1, both glutamate molecules remain in their binding sites. In contrast, during the Form 2 simulation, the glutamate in the open (T1R1) subunit consistently diffused away from its binding site, as illustrated in Fig. 3. To validate this conclusion, this simulation was repeated three times starting with different velocity profiles of the atoms at the beginning of the simulation, and analogous results were attained: glutamate migrated from the open binding site in Form 2, but not in Form 1. Thus, based on these simulation results, we propose that the active conformation of the umami receptor has the T1R1 ligand binding component in a closed conformation, and the T1R3 ligand binding domain in an open conformation. We note that
Zhang et al. recently modeled glutamate binding to T1R1 in the closed conformation [14]; however, they did not consider glutamate binding to T1R3. Our results are consistent with binding of glutamate to both T1R1 and T1R3 in the "closed-open/active" state, with T1R1 closed and T1R3 open (which we have termed Form 1), so our subsequent investigation centers on this Form 1 of the umami receptor.

\subsection{Binding sites}

Our molecular dynamics simulation of two glutamates bound to Form 1 shows a number of interactions to be important in the glutamate binding sites. These are shown graphically in Fig. 4 . The interactions were analyzed quantitatively by monitoring distances between glutamate and receptor atoms over successive 1 nanosecond periods during the simulation. Fig. 5 shows the normalized distribution function for a number of important distances in the T1R1 site during the course of the simulation, and Fig. 6 shows the normalized distribution function for distances in the T1R3 site.

In the T1R1 binding site, the glutamate $\alpha$-carboxylate group maintains a number of favorable interactions. There is a consistent ionic hydrogen bond interaction with both the backbone - $\mathrm{NH}$ and the sidechain $-\mathrm{OH}$ of Thr149. The distance from Thr149-N to the carboxylate carbon is centered at $3.69 \AA$ and the distance from Thr149-sidechain 0 to the carboxylate carbon is centered at $3.49 \AA$. The $\alpha$-carboxylate also binds to the backbone - $\mathrm{NH}$ of Ser172, with the
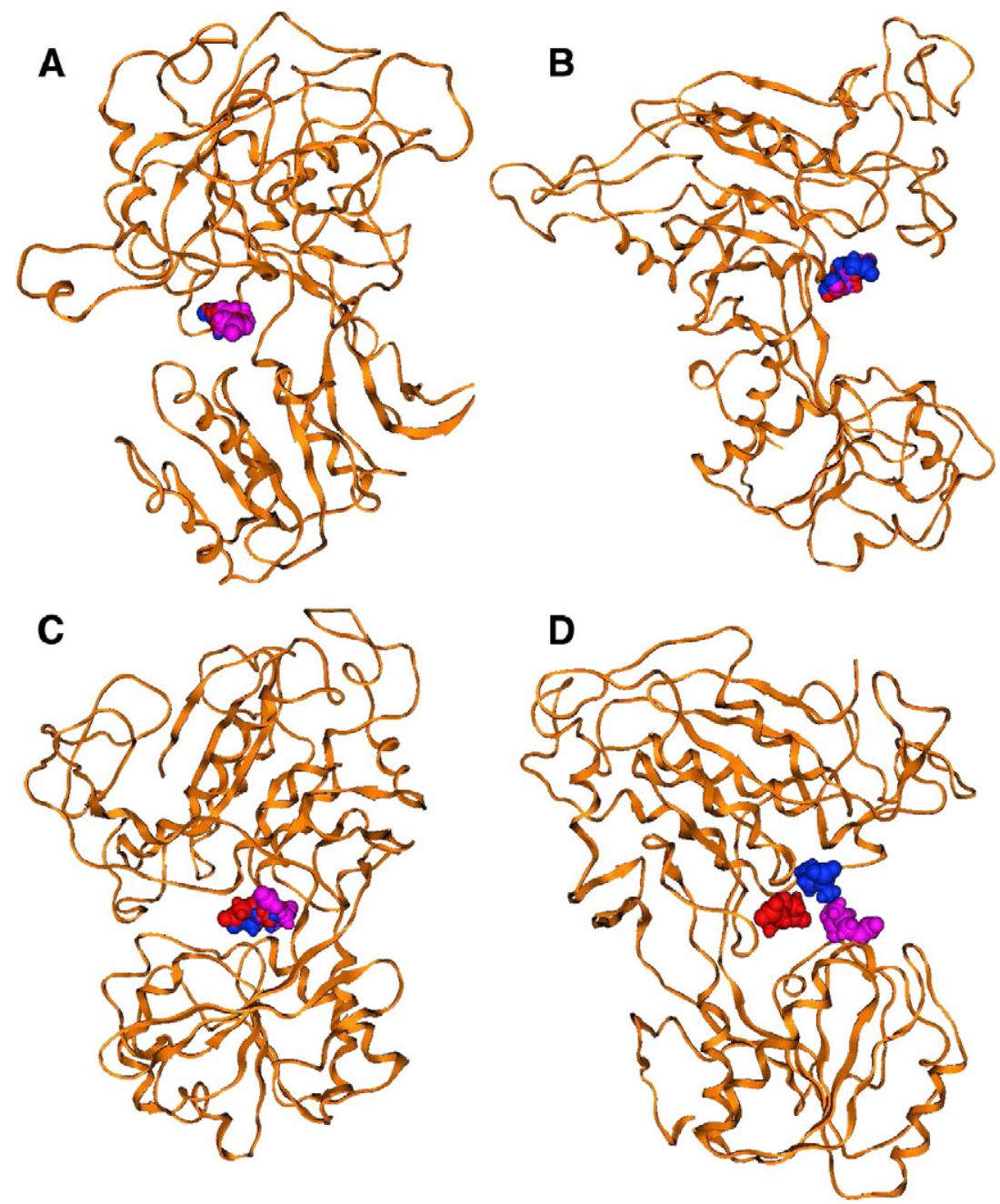

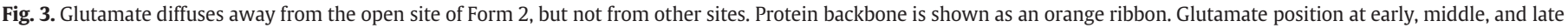

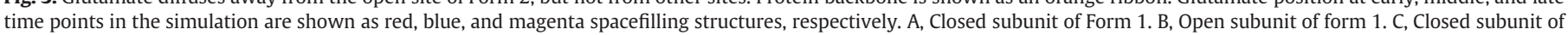
Form 2. D, Open subunit of Form 2. 

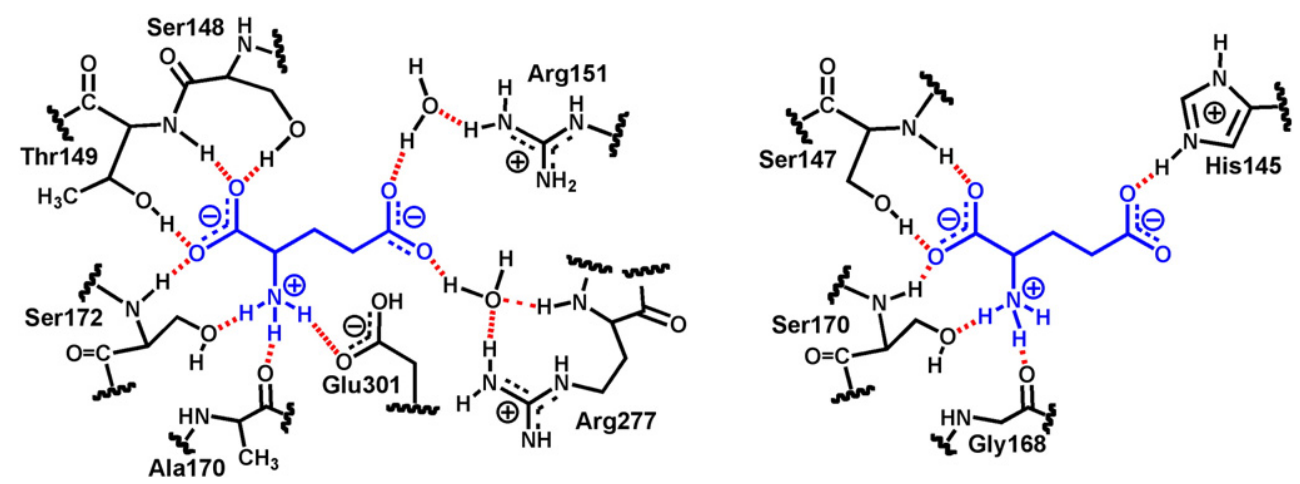

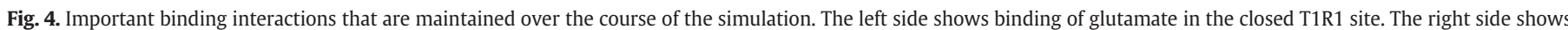
binding of glutamate in the open T1R3 site. The bound glutamate is shown in blue.

distance from Ser172-N to carboxylate carbon centered at $3.98 \AA$ A. In addition, there is an interaction with the sidechain $-\mathrm{OH}$ of Ser148 about $50 \%$ of the time; the distance distribution function shows peaks centered at 3.91 and $5.73 \AA$.

The glutamate amine nitrogen also maintains three favorable interactions, with the backbone-O of Ala170 (O-N distance centered at $2.81 \AA$ ), the sidechain-O of Ser172 (O-N distance centered at $2.91 \AA$ ), and the sidechain carboxylate of Glu301 (C-N distance centered at $3.34 \AA$ ). In contrast to the mGluR1 crystal structure, the amino group of the glutamate does not interact significantly with the aryl ring of Tyr220 (corresponding to Tyr235 in mGluR1) [4].

The $\gamma$-carboxylate of the glutamate is less firmly bound to this site. At different time points during the simulation, we can observe this carboxylate interacting either directly or through a bridging water molecule with the backbone - $\mathrm{NH}$ of Arg277, the sidechain guanidinium group of Arg277, and the sidechain guanidinium group of Arg151. These two arginines are sufficiently far apart that the carboxylate cannot interact with both simultaneously; it appears to alternately bind one, then the other. This is reflected in the distance distribution functions for these interactions. The distance between Arg277 backbone $\mathrm{N}$ and carboxylate carbon has peaks centered at $4.00 \AA$ and $6.60 \AA \AA$; the distance between $\operatorname{Arg} 277-C \zeta$ and carboxylate carbon has peaks centered at $3.87 \AA$, $4.29 \AA$, and $5.79 \AA$; the distance between Arg 151-C $\zeta$ and carboxylate carbon has a small peak centered at $4.62 \AA$ and a broad peak centered at $5.77 \AA$.

In the T1R3 binding site, the glutamate $\alpha$-carboxylate maintains favorable interactions with the backbone - NH groups of Ser147 and Ser170. The distance from Ser147-N to the carboxylate carbon is centered at $3.75 \AA$, and the distance from Ser170-N to the carboxylate

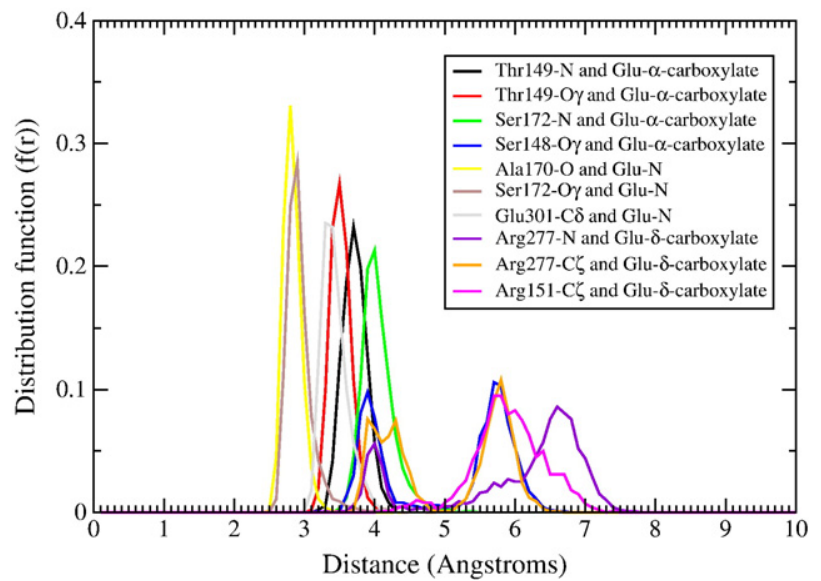

Fig. 5. Distance distribution functions for selected pairs of atoms between the closed T1R1 site and the bound glutamate. carbon is centered at $3.90 \AA$. The sidechain $-\mathrm{OH}$ of Ser 147 also interacts, with the distance from sidechain $\mathrm{O}$ to the carboxylate carbon centered at $3.41 \AA$.

The glutamate amine nitrogen maintains favorable interactions with the backbone carbonyl oxygen of Gly168 and the sidechain - $\mathrm{OH}$ of Ser170, with $\mathrm{O}-\mathrm{N}$ distances centered at $2.80 \AA$ and $2.90 \AA$, respectively. The glutamate gamma-carboxylate group maintains only a limited interaction with the His 145 sidechain. The distance from His145-Nع2 to the carboxylate carbon ranges from 3 to $5 \AA$.

Comparison to the mGluR1 structure [4] shows that binding of the $\alpha$-carboxylate and the $\alpha$-amino group is quite similar in the umami receptor, with serine and threonine backbone and sidechain groups playing important roles. This is consistent with the function of the umami receptor as a sensor for $\alpha$-amino acids. In contrast, the $\gamma$ carboxylate binding is substantially different between mGluR1 and the umami receptor. In mGluR1, the $\gamma$-carboxylate interacts with Lys409, Arg78, and (in the closed protomer) Arg323. In the closed T1R1 site, the $\gamma$-carboxylate interacts mainly with Arg277 through a water molecule bridge, and in the open T1R3 site, the $\gamma$-carboxylate interacts with His145. Since, in most species, the umami receptor is a fairly non-specific detector of $\alpha$-amino acids, it is not surprising that the ligand's side chain has fewer and less specific interactions than do the $\alpha$-carboxylate and the $\alpha$-amino groups.

\section{Binding free energy}

To understand the glutamate-receptor binding process, the relative free energies were estimated for the two glutamate binding sites of Form 1.

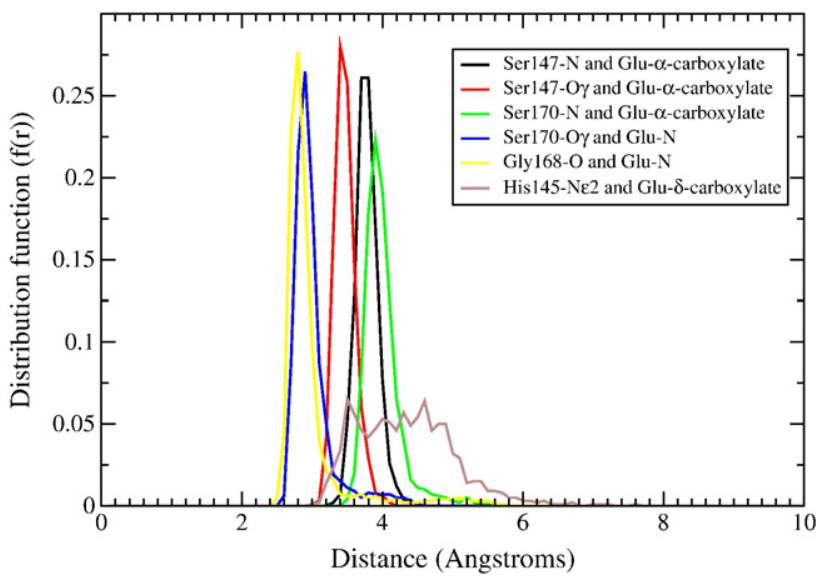

Fig. 6. Distance distribution functions for selected pairs of atoms between the open T1R3 site and the bound glutamate. 
For the studied association reaction $\mathrm{L}+\mathrm{P} \rightleftharpoons \mathrm{LP}$ between protein $\mathrm{P}$ and ligand $\mathrm{L}$ the potential of mean force as assessed by the umbrella sampling simulations [25] reflects the ratio of bound and unbound states as:

$\Delta G_{\text {bind }}=-k_{\mathrm{B}} T \ln \frac{[L P]}{[P]}$

where $k_{\mathrm{B}}$ is the Boltzmann constant and $\mathrm{T}$ the temperature. Thus, from calculating the potential of mean force (PMF) of the binding process, an estimation of the binding free energy i.e. of the affinity of glutamate for the binding sites of the T1R1 and T1R3 is obtained. We focused our efforts on evaluating the PMF for the two binding sites described earlier. We used the umbrella sampling method [25] to compute the PMF, where an artificial biasing potential is added to glutamate to force it to sample the regions of interest, from the binding site in the receptor to bulk solution. For convenience, the origin of the coordinate system was placed at the position of the glutamate after $100 \mathrm{~ns}$ of simulation, with the $z$-axis along the path away from the binding site. Negative values of $z$ represent movement deeper into the binding site, and positive values represent movement away from the receptor, into bulk solvent. For each binding site, 32 independent simulations of $30 \mathrm{~ns}$ each were performed. For each successive simulation, the glutamate molecule was shifted along the z-axis by $0.1 \mathrm{~nm}$, and the center of mass of the glutamate was restrained to that position by a harmonic potential on the z-axis, leaving it to move it freely in the xy plane. When the 32 simulations were completed, the weighted histogram analysis method (WHAM) [26] was used to obtain the PMF. The zero of free energy was placed in the bulk solution.

To confirm that the entropic contribution converged during our simulation times, a study of $\Delta G$ was performed for different simulation lengths. For simulations of $10 \mathrm{~ns}$, the free energy converged to the same values as for simulations of up to $30 \mathrm{~ns}$ length.

The free energy profile associated with the glutamate binding process was calculated, and Fig. 7 depicts the free energy profile associated with each binding site. The error bars were calculated after splitting the 30 ns of simulated trajectories into 3 sub-trajectories of 10 ns length.

From Fig. 7 was derived a minimum of $\Delta G$ of $-47.3 \mathrm{~kJ} / \mathrm{mol}$ associated with the binding site of T1R1, compared with the value of $-2.66 \mathrm{~kJ} / \mathrm{mol}$ obtained for binding site of T1R3. From these values of $\Delta G$, we conclude that the binding of glutamate to T1R1 is highly favorable whereas binding to T1R3 is expected to occur only transiently. We can find no experimental data for free energy of glutamate binding to taste receptors, to validate our simulations results. However, Morini et al. [9] report free energies for various sweeteners binding to the closely related T1R2/T1R3 sweet receptor,
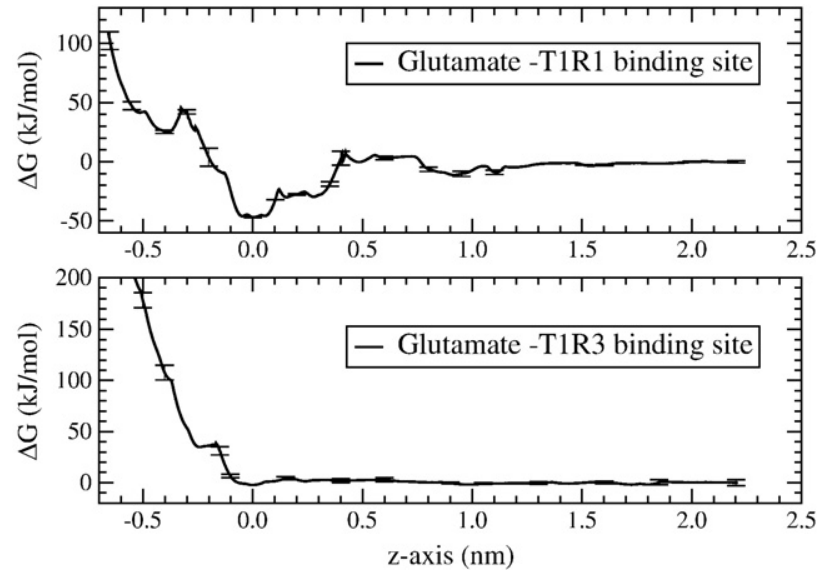

Fig. 7. Free Energy profile $(\Delta G)$ of binding glutamate to the T1R1 and T1R3 binding sites respectively. Error bars were calculated from 3 subtrajectories of 10 ns of simulation. with values ranging from -25 to $-57 \mathrm{~kJ} / \mathrm{mol}$, that are in line with our value calculated for T1R1.

\section{Conclusion}

Homology modeling and molecular dynamics simulation provide a model for the ways in which glutamate may interact with the umami taste receptor. We have identified probable binding sites and, at the atomic level, specific interactions likely to be involved in glutamate binding.

From thermodynamic calculations, the binding free energy of glutamate for the two binding sites was estimated. Binding to T1R1 was found to be highly favourable, whereas only transient binding to T1R3 would be expected based on our calculations.

\section{Acknowledgments}

J.J.L.C. acknowledges financial support from Fundación Seneca de la Región de Murcia through project 08647/PPC/08. The authors acknowledge the Computing Center of the Polytechnic University of Cartagena for their support and facilities to carry out the simulations on which this paper is based.

\section{Appendix A. Supplementary data}

Supplementary data to this article can be found online at doi:10. 1016/j.bpc.2010.09.001.

\section{References}

[1] X. Li, L. Staszewski, H. Xu, K. Durick, M. Zoller, E. Adler, Human receptors for sweet and umami taste, Proc. Natl. Acad. Sci. USA 99 (2002) 4692-4696.

[2] G. Nelson, J. Chandrashekar, M.A. Hoon, L. Feng, G. Zhao, N.J.P. Ryba, C.S. Zuker, An amino-acid taste receptor, Nature 416 (2002) 199-202.

[3] H. Jingami, S. Nakanishi, K. Morikawa, Structure of the metabotropic glutamate receptor, Curr. Opin. Neurobiol. 13 (2003) 271-278.

[4] N. Kunishima, Y. Shimada, Y. Tsuji, T. Sato, M. Yamamoto, T. Kumasaka, S. Nakanishi, H. Jingami, K. Morikawa, Structural basis of glutamate recognition by a dimeric metabotropic glutamate receptor, Nature 407 (2000) 971-977.

[5] D. Tsuchiya, N. Kunishima, N. Kamiya, H. Jingami, K. Morikawa, Structural views of the ligand-binding cores of a metabotropic glutamate receptor complexed with an antagonist and both glutamate and Gd3+, Proc. Natl. Acad. Sci. USA 99 (2002) 2660-2665.

[6] T. Muto, D. Tsuchiya, K. Morikawa, H. Jingami, Structures of the extracellular regions of the group II/III metabotropic glutamate receptors, Proc. Natl. Acad. Sci. USA 104 (2007) 3759-3764.

[7] M. Max, Y.G. Shanker, L. Huang, M. Rong, Z. Liu, F. Campagne, H. Weinstein, S. Damak, R.F. Margolskee, Tas1r3, encoding a new candidate taste receptor, is allelic to the sweet responsiveness locus sac, Nat. Genet. 28 (2001) 58-63.

[8] P.A. Temussi, Why are sweet proteins sweet? Interaction of brazzein, monellin and thaumatin with the T1R2-T1R3 receptor, FEBS Lett. 526 (2002) 1-4.

[9] G. Morini, A. Bassoli, P.A. Temussi, From small sweeteners to sweet proteins: anatomy of the binding sites of the human T1R2/T1R3 receptor, J. Med. Chem. 48 (2005) 5520-5520.

[10] P. Jiang, M. Cui, B. Zhao, Z. Liu, L.A. Snyder, L.M.J. Benard, R. Osman, R.F. Margolskee, M. Max, Lactisole interacts with the transmembrane domains of human T1R3 to inhibit sweet taste, J. Biol. Chem. 280 (2005) 15238-15246.

[11] P. Jiang, M. Cui, B. Zhao, L.A. Snyder, L.M.J. Benard, R. Osman, M. Max, R.F. Margolskee, Identification of the cyclamate interaction site within the transmembrane domain of the human sweet taste receptor subunit T1R3, J. Biol. Chem. 280 (2005) 34296-34305.

[12] D.E. Walters, G. Hellekant, Interactions of the sweet protein brazzein with the sweet taste receptor, J. Agric. Food Chem. 54 (2006) 10129-10133.

[13] W.F. van Gunsteren, H.J.C. Berendsen, Computer simulation of molecular dynamics: methodology, applications and perspectives in chemistry, Angew. Chem. Int. Ed Engl. 29 (1990) 992-1023.

[14] F. Zhang, B. Klebansky, R.M. Fine, H. Xu, A. Pronin, H. Liu, C. Tachdjian, X. Li, Molecular mechanism for the umami taste synergism, Proc. Natl. Acad. Sci. USA 105 (2008) 20930-20934.

[15] Y. Suzuki, E. Moriyoshi, D. Tsuchiya, H. Jingami, Negative cooperativity of glutamate binding in the dimeric metabotropic glutamate receptor subtype 1 , J. Biol. Chem. 279 (2004) 35526-35534.

[16] G.E. DuBois, Unraveling the biochemistry of sweet and umami tastes, Proc. Natl. Acad. Sci. USA 101 (2004) 13972-13973.

[17] H.J.C. Berendsen, J.P.M. Postma, W.F. van Gunsteren, J. Hermans, Intermolecular forces, in: B. Pullman (Ed.), Intermolecular Forces, Reidel, Dordrecht, 1981, p. 331. 
[18] E. Lindahl, B. Hess, D. van der Spoel, Gromacs 3.0: a package for molecular simulation and trajectory analysis, J. Mol. Model. 7 (2001) 306-317.

[19] H.J.C. Berendsen, D. van der Spoel, R. van Drunen, A message-passing parallel molecular dynamics implementation, Comp. Phys. Comm. 91 (1995) 43-56.

[20] W.L. Jorgensen, J. Tirado-Rives, The OPLS force field for proteins. Energy minimization for crystals of cyclic peptides and crambin, J. Am. Chem. Soc. 110 (1988) 1657-1666.

[21] H.J.C. Berendsen, J.P.M. Postma, W.F. van Gunsteren, A. DiNola, J.R. Haak, Molecular dynamics with coupling to an external bath, J. Chem. Phys. 8 (1984) 3684-3690.

[22] B. Hess, H. Bekker, H.J.C. Berendsen, H.J.C. Fraaije, Lincs: a linear constraint solver for molecular simulations, J. Comput. Chem. 18 (1997) 1463-1472.
[23] T. Darden, D. York, L. Pedersen, Particle mesh Ewald: an n.log(n) method for Ewald sums in large systems, J. Chem. Phys. 98 (1993) 10089-10092.

[24] U. Essmann, L. Perea, M.L. Berkowitz, T. Darden, H. Lee, L.G. Pedersen, A smooth particle mesh Ewald method, J. Chem. Phys. 103 (1995) 8577-8593.

[25] G.M. Torrie, J.P. Valleau, Nonphysical sampling distribution in Monte Carlo freeenergy estimation umbrella sampling, J. Comput. Phys. 23 (1977) 187-199.

[26] S. Kumar, J.M. Rosenberg, D. Bouzida, R.H. Swendsen, P.A. Kollman, The weighted histogram analysis method for free-energy calculations on biomolecules: I. The method, J. Comput. Chem. 13 (1992) 1011-1021. 